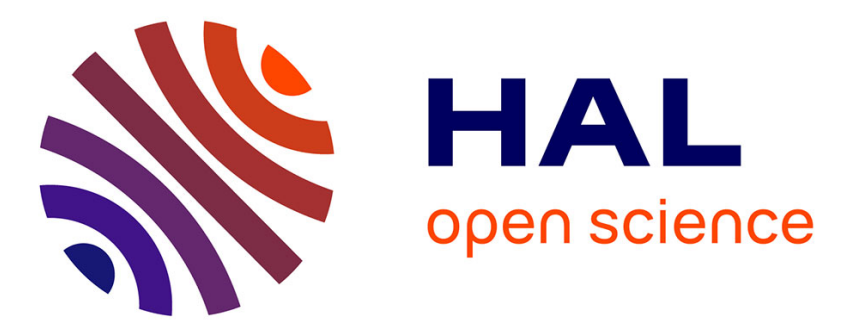

\title{
Mitochondrial respiratory mutants of Saccharomyces cerevisiae accumulate glycogen and readily mobilize it in a glucose-depleted medium
}

\author{
Brice Enjalbert, Jean Luc Parrou, Olivier Vincent, Jean Francois
}

\section{- To cite this version:}

Brice Enjalbert, Jean Luc Parrou, Olivier Vincent, Jean Francois. Mitochondrial respiratory mutants of Saccharomyces cerevisiae accumulate glycogen and readily mobilize it in a glucose-depleted medium. Microbiology, 2000, 146 (10), pp.2685-2694. 10.1099/00221287-146-10-2685 . hal-02146821

\section{HAL Id: hal-02146821 \\ https://hal.science/hal-02146821}

Submitted on 11 Jun 2019

HAL is a multi-disciplinary open access archive for the deposit and dissemination of scientific research documents, whether they are published or not. The documents may come from teaching and research institutions in France or abroad, or from public or private research centers.
L'archive ouverte pluridisciplinaire HAL, est destinée au dépôt et à la diffusion de documents scientifiques de niveau recherche, publiés ou non, émanant des établissements d'enseignement et de recherche français ou étrangers, des laboratoires publics ou privés. 


\title{
Mitochondrial respiratory mutants of Saccharomyces cerevisiae accumulate glycogen and readily mobilize it in a glucose-depleted medium
}

\author{
Brice Enjalbert, Jean Luc Parrou, Olivier Vincent $†$ and Jean François
}

Author for correspondence: Jean François. Tel: +33 5615594 92. Fax: +33561559400. e-mail: fran_jm@insa-tlse.fr

Centre de Bioingénierie Gilbert Durand, UMR-CNRS 5504, UR-INRA 792, Département de Génie Biochimique et Alimentaire, Complexe Scientifique de Rangueil, 31077 Toulouse, France

\begin{abstract}
Mutant strains of Saccharomyces cerevisiae defective in respiration have been reported to be unable to store glycogen, as revealed by the iodine-staining method. In this report, it is shown that in contrast to this claim, mitochondrial respiratory mutants accumulated even more glycogen than wild-type cells during the fermentative growth on glucose. However, as soon as glucose was exhausted in the medium, these mutants readily and completely mobilized their glycogen content, contrary to wild-type cells which only transiently degraded this polymer. The mobilization of glycogen was a specific trait resulting from a defect in mitochondrial function that could not be suppressed by mutations in the CAMP- and Pho85 protein kinase-dependent nutrientsensing pathways, and by other mutations which favour glycogen synthesis. To account for this mobilization, it was found that respiration-defective cells not only contained a less active glycogen synthase, but also a more active glycogen phosphorylase. Since glucose 6-phosphate (GIc6P) is a potent inhibitor of the phosphorylation and an activator of the dephosphorylation processes of glycogen synthase and glycogen phosphorylase, it is suggested that the drop in Glc6P observed at the onset of glucose depletion in respiration-deficient cells triggers this rapid and sustained glycogen mobilization. It is also proposed that this degradation provides the energy for the viability of respiratory mutants in glucose-starved medium.
\end{abstract}

Keywords: glycogen, trehalose, GSY2, respiration, Saccharomyces cerevisiae

\section{INTRODUCTION}

Glycogen is a polymer of glucosyl units linked by $\alpha-1,4$ bonds with $\alpha-1,6$ branches. It accounts for up to $10 \%$ of dry mass in yeast cells depleted for nitrogen, phosphorus and sulphur and at the end of growth on glucose-limited medium (Lillie \& Pringle, 1980). In the latter situation, glycogen accumulation is rapidly induced at the end of the exponential respiro-fermentative phase, while glucose and other nutrients are still plentiful in the medium (Parrou et al., 1999a). Glycogen deposition is also promoted by physico-chemical stresses, although to moderate levels due to a partial recycling of the polymer

† Present address: Department of Genetics and Development, Columbia University, USA.

Abbreviation: Glc6P, glucose 6-phosphate.
(Parrou et al., 1997). Conversely, the stored polymer is readily mobilized upon resumption of growth on a fresh medium or during spore germination. These rapid variations in the levels of glycogen in response to a variety of environmental changes highlight the complex regulatory patterns governing the metabolism of this polysaccharide (François et al., 1997).

Biochemical and genetic data accumulated over the last 10 years have unravelled at least two levels of control of glycogen metabolism in yeast. First, structural genes encoding enzymes involved in the biosynthesis and the biodegradation of glycogen are co-ordinately regulated under a wide variety of environmental conditions (Hwang et al., 1989; François et al., 1992; Thon et al., 1992; Hardy et al., 1994; Ni \& Laporte, 1995). In particular, the expression of these genes is co-induced when glucose concentration diminishes during diauxic 
Table 1. Genotypes of yeast strains used in this study

\begin{tabular}{|c|c|c|}
\hline Strain & Genotype & Source or reference \\
\hline JLP5-1A & a leu2 his3 ura3 URA3:: GSY2-lacZ & Parrou et al. (1999b) \\
\hline JF1069 & a leu2 his3 ura3 URA3:: GSY2-lacZ pet309:: Kan ${ }^{\mathrm{r}}$ & This study \\
\hline JF1233 & a leu2 his3 ura3 pho85: :HIS3 $\mathrm{RHO}^{+}$ & This study \\
\hline JF1234 & a leu 2 his 3 ura 3 pho $85:$ : HIS3 rho $^{\circ}$ & This study \\
\hline JLP48-3B & a ura3 URA3:: GSY2-lacZ $\mathrm{RHO}^{+}$ & Parrou et al. (1999b) \\
\hline JF1043 & a ura3 URA3:: GSY2-lacZ rho ${ }^{\circ}$ & This study \\
\hline JLP36-7B & $\begin{array}{l}\alpha \text { leu2 his3 ura3 URA3:: GSY2-lacZ ras2:: HIS3 } \\
\mathrm{RHO}^{+}\end{array}$ & Parrou et al. (1997) \\
\hline JF1242 & $\alpha$ leu 2 his 3 ura3 URA3:: GSY2-lacZ ras $2::$ HIS3 rho ${ }^{\circ}$ & This study \\
\hline JF624 & a leu2 his 3 ura3 lys 2 trp1 $\mathrm{RHO}^{+}$ & J. François \\
\hline JF1241 & a leu2 his 3 ura3 lys 2 trp1 rho ${ }^{\circ}$ & This study \\
\hline JF795 & $\alpha$ leu2 his 3 ura3 lys 2 trp1 gsy $1::$ URA3 gsy $2::$ HIS3 & J. François \\
\hline BWG1-7A & a leu2 his4 ade2 ura3 HAP2 & $\begin{array}{l}\text { B. Daignan-Fornier (IBGC- } \\
\text { CNRS, Bordeaux, France) }\end{array}$ \\
\hline J01-1A & a leu2 his4 ade2 ura3 hap2 & $\begin{array}{l}\text { B. Daignan-Fornier (IBGC- } \\
\text { CNRS, Bordeaux, France) }\end{array}$ \\
\hline W303-1A & a leu2 his3 ura3 trp1 ade2 QCR9 & $\begin{array}{l}\text { C. Gancedo (CSIC, } \\
\text { Madrid, Spain) }\end{array}$ \\
\hline JPD1 & a leu2 his3 ura3 trp1 ade2 qcr9:: URA3 & $\begin{array}{l}\text { C. Gancedo (CSIC, Madrid, } \\
\text { Spain) }\end{array}$ \\
\hline
\end{tabular}

growth by a mechanism which is strongly repressed by the RAS-cAMP pathway (Hardy et al., 1994; Parrou et al., 1999b). A second level of control involves covalent modification by reversible phosphorylation of glycogen phosphorylase (Gph1p) and glycogen synthase (Gsy2p) which leads to the activation of the former and the inactivation of the latter. A cluster of three phosphorylation sites at the $\mathrm{COOH}$ terminus of Gsy2p has been identified and site-directed mutagenesis of these sites or deletion of a $61 \mathrm{COOH}$ terminus fragment blocks Gsy2p in a hyperactivated form (Hardy \& Roach, 1993). Two protagonists implicated in the control of the Gsy2p phosphorylation state have been characterized: a glycogen synthase kinase consisting of the Pho85pPcl10/Pcl8 complex (Huang et al., 1997; Wilson et al., 1999) and a glycogen synthase phosphatase corresponding to the Glc7p-Gac1p complex (François et al., 1992; Skroch-Stuart et al., 1994). In contrast, nothing is known about the protein kinase(s) and protein phosphatase(s) regulating the phosphorylation state of glycogen phosphorylase. Evidence from several experiments demonstrates that glucose 6-phosphate (Glc6P) is a potent activator of the dephosphorylation and an inhibitor of the phosphorylation processes mediated by these protagonists (François \& Hers, 1988; Lin et al., 1996; Huang et al., 1997). In addition, Glc6P allosterically stimulates glycogen synthase and inhibits glycogen phosphorylase (reviewed by François et al., 1997).

The iodine-staining reaction has been widely used to search for mutants affected in glycogen metabolism, since yeast colonies growing on agar plates develop a brown coloration proportional to their glycogen content upon exposure to iodine crystal vapour (Chester, 1968). This method led to the identification of mutants with a strong glycogen phenotype, e.g. no glycogen or hyperaccumulation of this polymer. Hence, mutations in components of the Ras-cAMP signalling pathway, in SNF1 or PHO85 genes encoding key protein kinases directly or indirectly implicated in carbon metabolism, were often uncovered (Thompson-Jaeger et al., 1991; Wek et al., 1992; Cannon et al., 1994; Huang et al., 1996; Timblin et al., 1996). Interestingly, some isolated glycogen-deficient mutants were also defective in respiration and presented a petite phenotype (Chester, 1968; Filipak et al., 1992; Yang et al., 1998). This linkage between mitochondrial function and glucose storage was recently investigated at the genetic level by Yang et al. (1998). These authors suggested that the inability of mitochondrial respiratory mutants to synthesize glycogen was due to the inactivation of glycogen synthase by a Ras-cAMP-dependent, Pho85pindependent mechanism.

As part of our work aiming to identify the mechanism of the early induction of glycogen synthesis during diauxic growth on glucose, we sought mutants defective in this process using a double genetic screen based on the lack of iodine staining of colonies and loss of $\beta$-galactosidase activity from a GSY2-lacZ construct. Among the isolated mutants, we observed that one of them, which was respiration-deficient, accumulated glycogen during growth on glucose despite its negative iodine-staining reaction. Hence, the discrepancy between the qualitative iodine-staining assay and the quantitative kinetic analysis of glycogen was further investigated.

\section{METHODS}

Yeast strains, plasmids and growth conditions. The strains used in this study are listed in Table 1. The gsy1::URA3 
gsy2:: HIS3 mutant (strain JF795) was obtained after six backcrosses between JF624 and IF3 from Farkas et al. (1991). The pet309:: $\operatorname{Kan}^{\mathrm{r}}$ construct in plasmid pEB309Kan was obtained

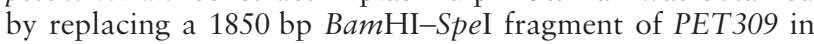
pEB309 (cf. following section for description of pEB309), with a $1.5 \mathrm{kbp}$ BamHI-SpeI KanMX4 fragment from pFA6akanMX4 (Wach et al., 1994). Disruption of PET309 in strain JLP5-1A was performed using the $2213 \mathrm{bp} \mathrm{MscI-MscI}$ pet309:: $\mathrm{Kan}^{\mathrm{r}}$ disruption cassette from pEB309Kan. Disruption of PHO85 in strain JLP5-1A was performed using the $3.1 \mathrm{kbp}$ SalI-BamHI pho85::HIS cassette from p1885SX:: HIS3 (Timblin et al., 1996). pGSY2AC, a high-copynumber vector (kind gift of P. Roach; Hardy \& Roach, 1993), was used to transform the wild-type JF624 and its $r h o^{\circ}$ derivative. Transformation of yeast strains was carried out using the lithium acetate method of Schietsl \& Gietz (1989). Yeast cells were grown in a rich medium (YEPD) containing $10 \mathrm{~g}$ Yeast Extract, $20 \mathrm{~g}$ Bacto peptone and $10 \mathrm{~g}$ glucose $\mathrm{l}^{-1}$, or in a synthetic minimal medium (YNB) containing $0.17 \%$ $(\mathrm{w} / \mathrm{v})$ yeast nitrogen base without amino acids and ammonium, $0.5 \%(\mathrm{w} / \mathrm{v})$ ammonium sulphate and $1 \%(\mathrm{w} / \mathrm{v})$ glucose. Unless otherwise stated, cultures were grown at $30^{\circ} \mathrm{C}$ in 21 shake flasks containing 0.31 culture medium. Cell growth was followed at $\mathrm{OD}_{600}$.

Isolation of respiration-deficient cells. UV mutagenesis was carried out with strain JLP5-1A to generate glycogen-deficient mutants according to the procedure of Chester (1968). Among 90 isolates which coloured yellow upon exposure to iodine vapour after $3 \mathrm{~d}$ growth on YEPD agar plates, 15 of them were also faint blue or white after $\beta$-galactosidase assay on permeabilized cells (Guthrie \& Fink, 1991). From this set of mutants, one harboured typical traits of respiration-deficient cells (small size of colonies and inability to grow on acetate). After two back-crosses with the original wild-type, this mutant was transformed with a yeast genomic library inserted into YCp50 (purchased from American Type Culture Collection, Manassas, VA, USA; ATCC 37415) to screen for clones that restore glycogen as checked by iodine staining. Plasmids rescued from independent clones contained overlapping genomic fragments encompassing the PET309 gene. A 3325 bp NruI-BglII fragment containing only PET309 was subcloned into NruI-BamHI sites of YCp50 to yield pEB309. Transformation with this plasmid restored glycogen accumulation in the petite mutant as checked by iodine staining. To obtain rho ${ }^{\circ}$ cells, strains JLP48-3B, JLP36-7B, JF1233 and JF624 were treated with ethidium bromide, according to the procedure of Fox et al. (1991), and petite strains were identified by their inability to grow on non-fermentable carbon sources.

Biochemical and analytical procedures. Preparation of extracts and assay of glycogen phosphorylase and glycogen synthase were carried out as described by François et al. (1988). For glycogen synthase, the assay was done using $0.25 \mathrm{mM}$ UDP-Glc as the substrate, in the absence or presence of $20 \mathrm{mM}$ Glc6P. The activation state (or non-phosphorylated state) of this enzyme is estimated by the \pm Glc6P activity ratio of glycogen synthase. Preparation of extracts, measurement of $\beta$-galactosidase activity and determination of glycogen and trehalose were performed as described by Rose \& Botstein (1993) and Parrou \& François (1997). Qualitative assessment of glycogen was carried out by using the iodine-staining method (Chester, 1968) with cells spotted on YEPD (rich) or yeast nitrogen base complemented with auxotrophic requirement (minimal) agar plates. At different times, the plates were inverted over iodine crystals for $1 \mathrm{~min}$, removed for $15 \mathrm{~s}$ and exposed again for $2 \mathrm{~min}$. To obtain reliable results, a different plate was used for each staining to avoid any possible growth interference induced by the iodine vapour. To assay Glc6P and nucleotides, the rapid quenching method of yeast cells and extraction of metabolites in boiling buffered ethanol was followed (Gonzalez et al., 1997). Determination of Glc6P, ATP and ADP was performed by coupling with NADH production or consumption as described by Bergmeyer (1986). Results reported in Figs 1, 2 and 4 are from one set of experiments repeated independently two times.

\section{RESULTS}

\section{Respiratory mutants are not impaired in glycogen accumulation during the growth on glucose-limiting medium}

The early co-induction of genes involved in glycogen metabolism during diauxic growth on glucose (Parrou

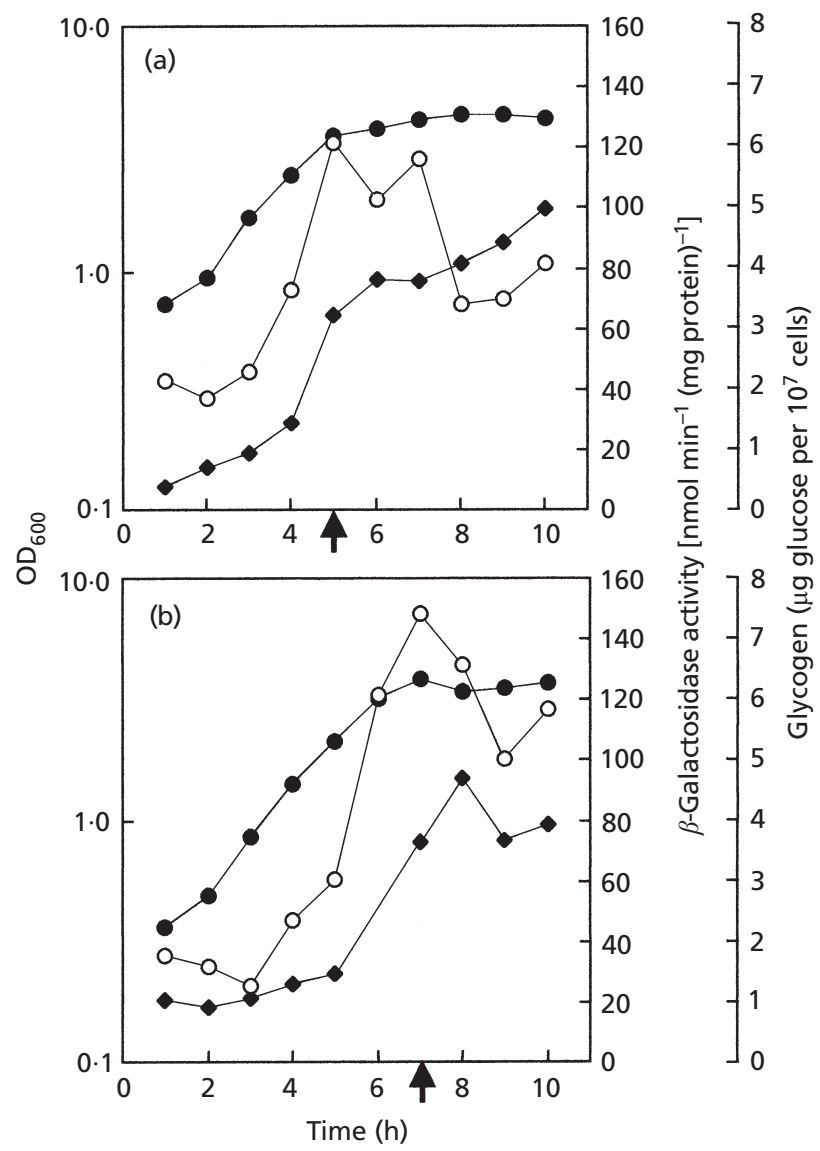

Fig. 1. Kinetics of glycogen accumulation and $\beta$-galactosidase levels from a GSY2-lacZ construct in a pet309 mutant and its isogenic wild-type strain during respiro-fermentative growth on glucose-limiting medium. Control strain JLP5-1A (a) and the pet309 mutant JF1069 (b) were cultivated in a 1 I shake-flask containing $300 \mathrm{ml}$ YEP with $10 \mathrm{~g}$ glucose $\mathrm{I}^{-1}$. $\square$, OD; $O$, glycogen; $\diamond, \beta$-galactosidase. The arrows indicate the time of glucose exhaustion in the medium. 


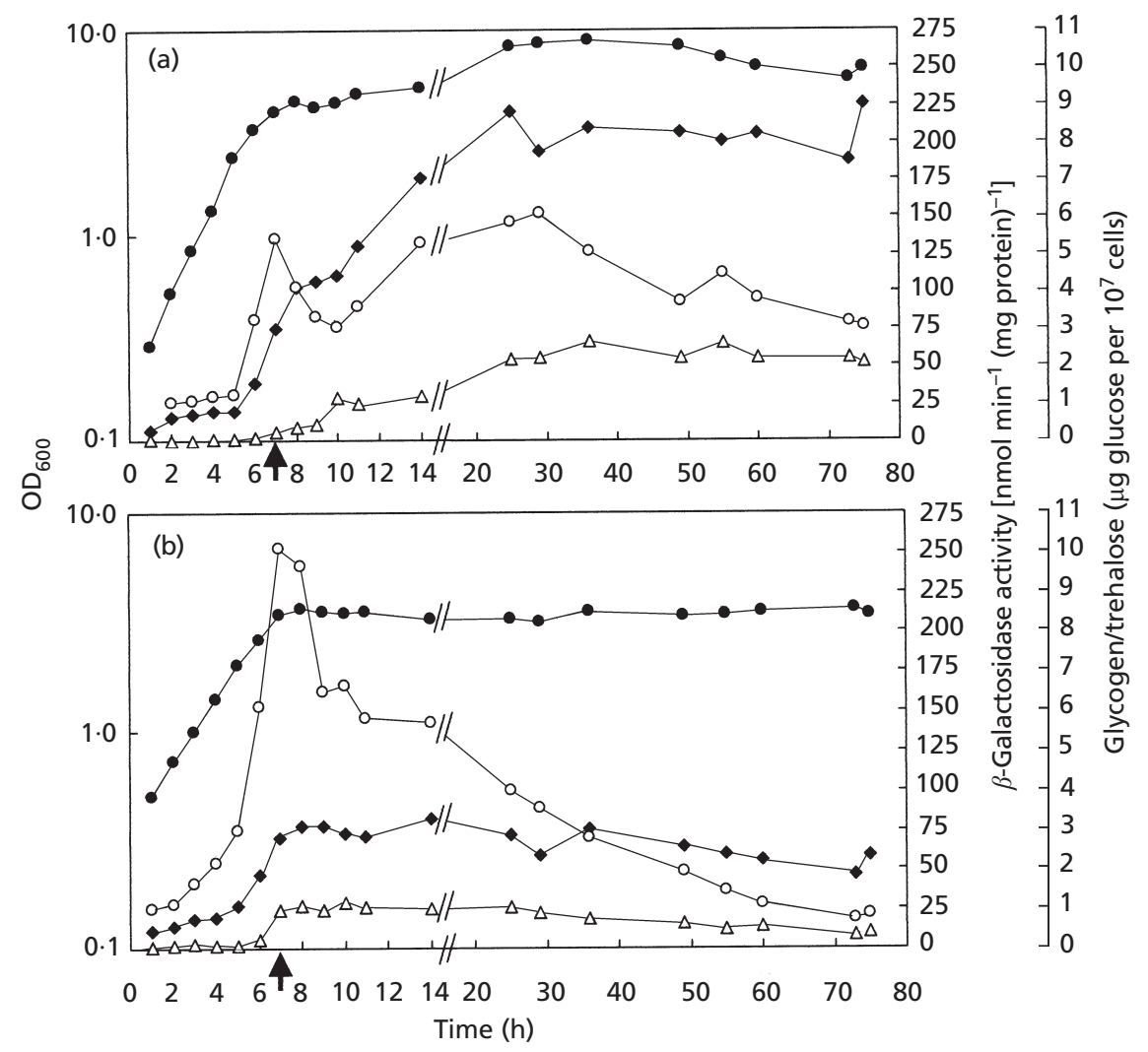

Fig. 2. Kinetics of glycogen and $\beta$-galactosidase levels from a GSY2-lacZ construct in a rho ${ }^{\circ}$ mutant and its isogenic wildtype strain during long-term culture on a glucose-rich medium. Control strain JLP48-3B (a) and rho ${ }^{\circ}$ strain JF1043 (b) were cultivated in a 21 batch fermenter containing $1.7 \mathrm{I}$ YEP with $10 \mathrm{~g}$ glucose $\mathrm{I}^{-1} .0, \mathrm{OD} ; \mathrm{O}, \mathrm{glycogen;} \triangle$, trehalose; $\bullet, \beta$ galactosidase. The arrows indicate the time of glucose exhaustion in the medium.

et al., 1999a) led us to initiate a genetic screen to characterize this specific event. After UV mutagenesis, mutants with reduced glycogen content were isolated based on the loss of iodine staining and of $\beta$-galactosidase expression from a GSY2-lacZ construct. This resulted in the isolation of clones with mutations in the RAS-cAMP pathway while another displayed typical traits of a petite strain. The latter was complemented by a genomic fragment containing PET309, encoding a nuclear gene necessary for the maturation of the mitochondrial COX1 messenger (Manthey \& McEwen, 1995). Disruption of PET309 confirmed that the loss of function of this gene led to reduced glycogen accumulation, as estimated by iodine staining of cell patches after $3 \mathrm{~d}$ growth on YNB-agar plates. However, in contrast with the iodine-staining results, glycogen accumulation and $\beta$-galactosidase activity from the GSY2-lacZ construct were not significantly affected during the fermentative growth of a pet309 mutant on glucose (Fig. 1).

This unexpected result prompted us to investigate whether the pattern of glycogen accumulation was specific to the pet309 mutation or was a general feature of cells defective in mitochondrial respiration. For this purpose, we used petite cells lacking mitochondrial DNA (rho ${ }^{\circ}$ mutant) which accumulated even more glycogen than wild-type cells at the end of growth on glucose (Fig. 2). At the onset of glucose exhaustion, glycogen started to be mobilized in both wild-type and petite cells. This mobilization was only transient in wild-type cells, whereas it was sustained in the $r h o^{\circ}$ mutant such that it contained four times less glycogen than the wild-type after $3 \mathrm{~d}$ in glucose-starved medium. Fig. 2 also shows that the activity of $\beta$-galactosidase from the GSY2-lacZ construct in the $r o^{\circ}$ strain was $30 \%$ lower than that in the wild-type at the end of growth on glucose. This activity remained unchanged during prolonged incubation in the glucose-depleted medium, while it increased in the wild-type by about two times, indicating a resumption of the transcriptional activity during the respiratory phase of growth on ethanol. Another notable difference between wild-type and respiration-deficient cells was that the latter contained very low levels of trehalose. In both types of cells, the accumulation of trehalose was initiated at the moment when a minute amount of glucose remained in the growth medium (Parrou et al., 1999a; Fig. 2). Unlike wild-type cells, which accumulated the disaccharide during the respiratory growth phase on ethanol, tre- 


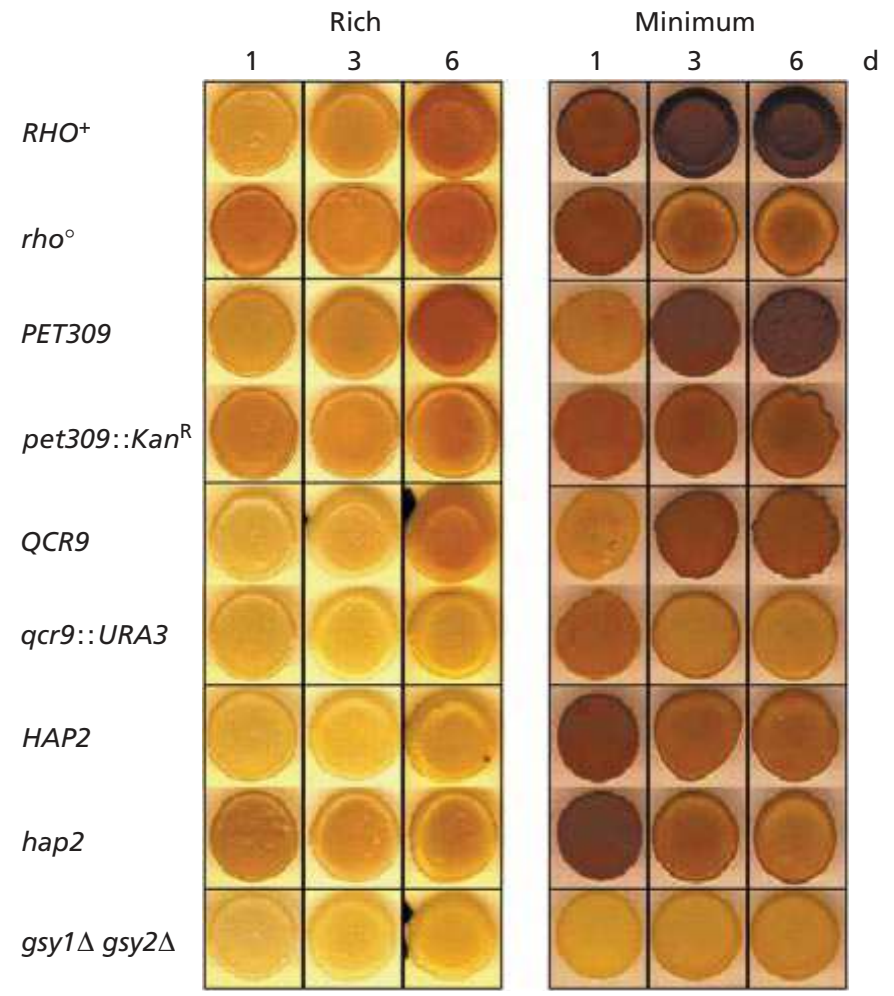

Fig. 3. Exposure of wild-type cells and mitochondrial respiration-deficient cells to iodine vapour at different times of growth on agar plates. Strains were grown for $2 \mathrm{~d}$ on YEPD solid medium and then $10 \mu \mathrm{l}$ was spotted on YEPD (Rich) or YNB (Minimal) agar plates complemented with auxotrophic requirements. Exposure to iodine vapour was done after 1,3 or $6 \mathrm{~d}$ growth using a different plate for each exposure. The following strains were used: $\mathrm{RHO}^{+}$(JLP483BA), rho ${ }^{\circ}$ (JF1043), PET309 (JLP5-1A), pet309:: $\operatorname{Kan}^{\mathrm{R}}$ (JF1069), QCR9 (W303-1A), qcr9::URA3 (JPD1), HAP2 (BWG1-7A), hap2

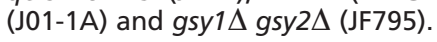

halose synthesis was stopped in the $r h o^{\circ}$ mutant as soon as exogenous glucose was consumed.

\section{Time-course iodine staining of respiratory mutants correlates with glycogen content in liquid cultures}

As shown in Figs 1 and 2, the high level of glycogen accumulation in mitochondrial respiratory mutants during growth on glucose contrasted with their reported lack of iodine staining on agar plates (Chester, 1968; Wek et al., 1992; Cannon et al., 1994; Yang et al., 1998). To test the reliability of the iodine-staining method, we performed time-course coloration of cell patches of wild-type and respiratory mutants altered in genes involved in respiratory function (Tzagaloff \& Dieckmann, 1990). In close correlation with quantitative glycogen assays, pet309 and rho mutants turned even more brown than isogenic wild-type when the staining was performed $1 \mathrm{~d}$ after spotting on agar plates (Fig. 3). Similar results were obtained with strains bearing mutations in QCR9, encoding a subunit of cytochrome $b c 1$, and HAP2, encoding a nuclear transcriptional factor involved in global regulation of respiratory genes. In contrast, the mitochondrial respiratory mutants exhibited a lower iodine staining after $3 \mathrm{~d}$ growth and this reduced coloration was even more severe after $6 \mathrm{~d}$. This feature was observed on both rich and minimal agar plates, despite better iodine staining, and hence provides a contrast between wild-type and respiratory mutant strains on minimal plates. As a control, the gsy1s gsy $2 \Delta$ double mutant which is defective in glycogen synthase activity (Farkas et al., 1991) remained yellow throughout the experiment. Quantitative measurement confirmed the iodine-staining results since glycogen content was three- to fivefold lower in the mitochondrial respiratory mutants than in their isogenic control after $2 \mathrm{~d}$ growth in liquid medium (Table 2). We conclude from these experiments that a time-course iodine staining of yeast colonies is absolutely required to obtain confident data on mutations affecting glycogen metabolism.

\section{Mobilization of glycogen in mitochondrial respiratory mutants is not abolished in pho85 and ras2 mutants}

Since the Ras-cAMP pathway and the cyclin-dependent Pho85 protein kinase play an important role in the control of glycogen metabolism (François et al., 1997; Huang et al., 1996; Timblin et al., 1996), we investigated whether a mutation in PHO85 or RAS2 would alter the mobilization of glycogen during longterm incubation of respiratory mutants in glucosestarved media. Consistent with our previous finding in Fig. 3, a $r h o^{\circ}$ derivative of the pho85 mutant cultivated on rich medium mobilized its glycogen store even faster than the $\mathrm{PHO} 85 \mathrm{rbo}^{\circ}$ strain as soon as glucose was exhausted in the medium (cf. Figs $2 \mathrm{~b}$ and $4 \mathrm{~b}$ ), whereas it remained high in pho85 $\mathrm{RHO}^{+}$cells during their slow resumption of growth on ethanol (Fig. 4). Similar results were found in a $r h o^{\circ}$ derivative of the ras 2 mutant during prolonged incubation in glucose-depleted liquid medium (not shown). These glycogen patterns in liquid cultures grossly correlated with the time-course iodine 
Table 2. Levels of glycogen and activity of glycogen synthase and phosphorylase in various respiration-deficient mutant strains and their isogenic wild-type as determined about $24 \mathrm{~h}$ after glucose exhaustion in the medium

Yeast cells were grown on YEP containing 1\% glucose. Cells were harvested about $24 \mathrm{~h}$ after complete exhaustion of glucose in the medium. Measurements of glycogen and enzymic activities were performed as described in Methods. Values reported are duplicates from three independent experiments.

\begin{tabular}{|c|c|c|c|c|c|c|}
\hline \multirow[t]{2}{*}{ Strain } & \multirow[t]{2}{*}{ Genotype } & \multirow{2}{*}{$\begin{array}{c}\text { Glycogen }(\mu \mathrm{g} \\
\text { glucose per } \\
\left.10^{7} \text { cells }\right) \\
\pm \text { SD }\end{array}$} & \multicolumn{3}{|c|}{$\begin{array}{l}\text { Glycogen synthase }\left[\mathrm{nmol} \mathrm{min}^{-1}\right. \\
\left.(\mathrm{mg} \text { protein })^{-1} \pm \mathrm{SD}\right]\end{array}$} & \multirow{2}{*}{$\begin{array}{c}\text { Glycogen } \\
\text { phosphorylase } \\
{\left[\text { nmol min }{ }^{-1}\right.} \\
\left.(\text { mg protein })^{-1} \pm S D\right]\end{array}$} \\
\hline & & & - Glc6P & + Glc6P & Ratio (\%) & \\
\hline JLP48-3B & $\mathrm{RHO}^{+}$ & $5 \cdot 5 \pm 0 \cdot 50$ & $11 \cdot 1 \pm 0 \cdot 5$ & $14 \cdot 2 \pm 2 \cdot 4$ & 78 & $14 \cdot 5 \pm 2 \cdot 3$ \\
\hline JF1043 & {$\left[r h o^{\circ}\right]$} & $3 \cdot 4 \pm 0 \cdot 30$ & $3 \cdot 10 \pm 0 \cdot 5$ & $13 \cdot 7 \pm 1 \cdot 9$ & 22 & $28 \cdot 5 \pm 2 \cdot 5$ \\
\hline JLP5-1A & РET309 & $4 \cdot 9 \pm 0 \cdot 45$ & $8 \cdot 2 \pm 0 \cdot 9$ & $13 \cdot 4 \pm 2 \cdot 0$ & 62 & $22 \cdot 4 \pm 2 \cdot 6$ \\
\hline JF1069 & pet309::Kan ${ }^{\mathrm{r}}$ & $1 \cdot 5 \pm 0 \cdot 15$ & $2 \cdot 0 \pm 0 \cdot 4$ & $10 \cdot 3 \pm 1 \cdot 6$ & 19 & $48 \cdot 6 \pm 8 \cdot 8$ \\
\hline W303-1A & QCR9 & $7 \cdot 20 \pm 0 \cdot 55$ & $11 \cdot 9 \pm 4 \cdot 6$ & $16 \cdot 5 \pm 5 \cdot 0$ & 72 & $22 \cdot 8 \pm 6 \cdot 8$ \\
\hline JPD1 & qcr $9:: U R A 3$ & $2 \cdot 0 \pm 0 \cdot 25$ & $6 \cdot 2 \pm 1 \cdot 0$ & $18 \cdot 2 \pm 2 \cdot 2$ & 32 & $38 \cdot 9 \pm 7 \cdot 9$ \\
\hline BWG1-7A & HAP2 & $6 \cdot 5 \pm 0 \cdot 50$ & $4 \cdot 3 \pm 0 \cdot 5$ & $6 \cdot 6 \pm 0 \cdot 9$ & 80 & $40 \cdot 5 \pm 11 \cdot 7$ \\
\hline JO1-1A & hap2 & $0 \cdot 75 \pm 0 \cdot 10$ & $2 \cdot 5 \pm 0 \cdot 5$ & $6 \cdot 1 \pm 0 \cdot 9$ & 40 & $67 \cdot 9 \pm 12 \cdot 0$ \\
\hline
\end{tabular}

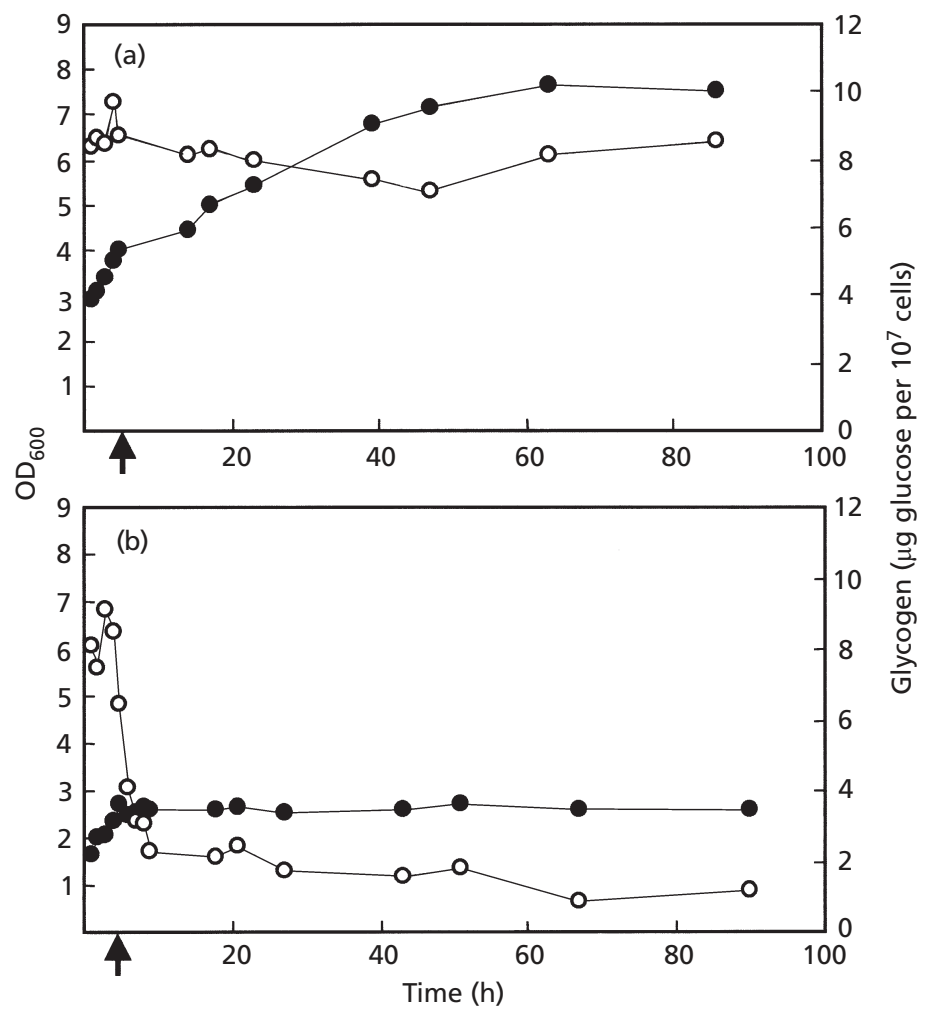

Fig. 4. Kinetics of glycogen in a pho85 mutant and its $r h 0^{\circ}$ derivative during the respiratory growth phase on glucose medium. The pho85 mutant (strain JF1233; a) and the pho85 $r$ o ${ }^{\circ}$ mutant (strain JF1234; b) were cultivated in a 1 I shake-flask containing $300 \mathrm{ml}$ YEP with $10 \mathrm{~g}$ glucose $\mathrm{I}^{-1}$., $\mathrm{OD} ; \mathrm{O}$, glycogen. The arrow indicates the time of glucose exhaustion in the medium.

staining on YEPD agar plates as shown in Fig. 5(a). However, the rate of glycogen degradation upon prolonged incubation of respiration-deficient cells on minimal agar plates was diminished by mutations that led to a reduction of cAPK or Pho85p activity. These results indicate that mutations in these two main nutrientsensing pathways do not abolish the ability of respiration-deficient cells to mobilize glycogen, but clearly affect its rate of degradation depending on the growth medium.

\section{The activated form of Gsy2p does not override glycogen mobilization in respiratory mutant strains}

Based on iodine-staining results, Yang et al. (1998) showed that the glycogen defect in mitochondrial 
(a)

Control

pho85:: HIS3

pho85:: HIS3 rho ${ }^{\circ}$

$\operatorname{ras} 2::$ HIS3

ras2::HIS3 $r h 0^{\circ}$
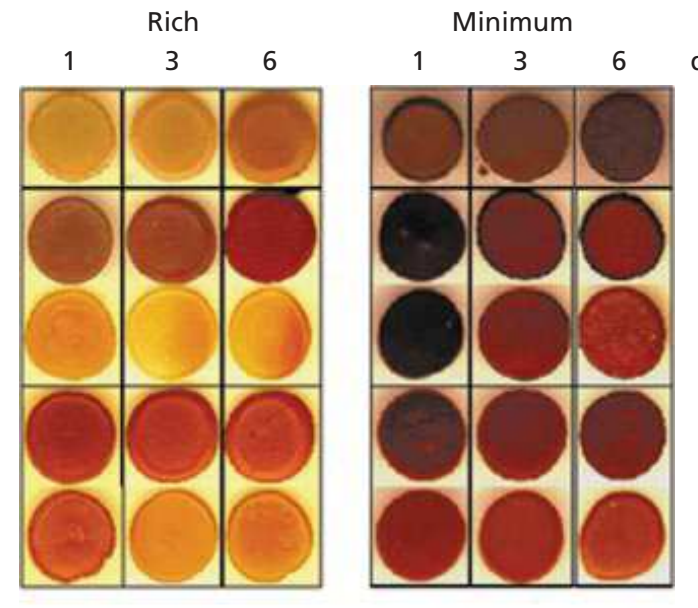

(b)

$\mathrm{RHO}^{+} / \mathrm{pRS} 314$

$r h o \%$ pRS314

$R H O^{+} / p G S Y 2 \Delta C$

$r h o \%$ pGSY $2 \Delta \mathrm{C}$

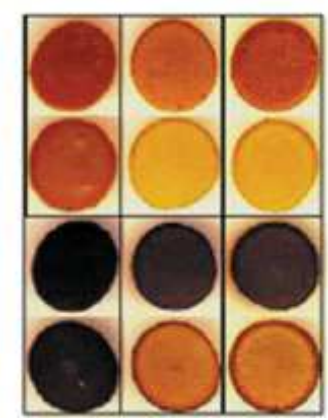

d

Fig. 5. Exposure to iodine vapour of ras2 and pho85 mutants and their $r \mathrm{r}^{\circ}$ derivatives (a) and a wild-type strain and its $r h o^{\circ}$ derivative expressing a hyperactivated form of glycogen synthase (b) at different times of growth on agar plates. Strains were grown for $2 \mathrm{~d}$ on YEPD solid medium and then $10 \mu \mathrm{l}$ was spotted on YEPD (Rich) or YNB (Minimal) agar plates complemented with auxotrophic requirements. Exposure to iodine vapour was made after 1, 3 or $6 \mathrm{~d}$ growth using a different plate for each exposure. In (a) the mutant strains used were all derived from the control JLP5-1A (pho85, JF1233; pho85 rho ${ }^{\circ}$, JF1234; ras2, JLP36-7B; ras2 rho $^{\circ}$, JF1242). In (b) the wildtype strain JF624 or its $r \mathrm{ro}^{\circ}$ derivative JF1241 were transformed with the control vector (pRS314) and with $\mathrm{pGSY} 2 \Delta \mathrm{C}$ which expresses a truncated activated form of Gsy2p. respiratory mutants was suppressed by the expression of a constitutively activated form of glycogen synthase. Since we demonstrated that these mutants are not impaired in glycogen synthesis but readily mobilize the polymer during long-term incubation after glucose exhaustion, we investigated whether the presence of an activated form of Gsy2p (GSY2 2 C construct) in petite cells may interfere with glycogen mobilization. It is shown in Fig. 5(b) that, as expected, glycogen deposition was strongly enhanced in wild-type and $r h o^{\circ}$ strains expressing the GSY $2 \Delta C$ construct, as illustrated by the dark blue coloration of the cells upon iodine vapour treatment after $1 \mathrm{~d}$ growth. However, as judged by iodine staining, the mobilization of glycogen in transformed $r h o^{\circ}$ cells upon prolonged incubation on YEPD agar plates was apparently not significantly greater than that in the transformed control cells. It is possible that this apparent absence of glycogen degradation is a consequence of its very high initial content in these cells at the limit of the iodine-staining method. By comparison, the sharp drop in iodine staining after 3 and $6 \mathrm{~d}$ growth of $r h o^{\circ}$ cells expressing GSY $2 \Delta C$ on minimal agar plates indicated a stronger requirement of respiration-deficient cells cultivated on poor growth media for their glycogen store, as observed in Fig. 3. Our results are therefore at variance with those of Yang et al. (1998). Although no clear explanation can be offered for this discrepancy, it must be recalled that a single time point measurement of glycogen content by the iodinestaining method may lead to misleading conclusions.

\section{Defect in respiration stimulates glycogen mobilization by a lowering of Glc6P}

The sustained degradation of glycogen in mitochondrial respiratory mutants, particularly in a $r h o^{\circ}$ strain expressing a hyperactivated form of glycogen synthase in minimal medium, supports the idea that the glycogen degradation pathway is stimulated in addition to the inhibition of Gsy2p-linked biosynthesis. In agreement with this suggestion, it is shown in Table 2 that the glycogen phosphorylase activity was $1 \cdot 5$-to 3 -fold higher in the respiratory mutants. The activation state of glycogen synthase in respiratory mutants was three to six times lower than that measured in wild-type cells (Yang et al., 1998; Table 2). These enzymic changes were correlated with a sharp drop in Glc6P pools measured at the onset of glucose depletion (Table 3) and remained at low levels during prolonged incubation (not shown). That the Glc6P pool was very low may be due to the fact that gluconeogenesis is not operative in these mutants. The intracellular concentrations of ATP and ADP were also lower than in wild-type cells, presumably due to the failure of these cells to shift to a respiratory mode of growth. Since Glc6P is a key effector in the control of the phosphorylation state of the two enzymes (François \& Hers, 1988; Lin et al., 1997; Huang et al., 1997), it is suggested that glycogen mobilization may be triggered by the drop in the level of this sugar phosphate observed in the respiratory mutants after glucose depletion. 
Table 3. Levels of Glc6P, ATP and ADP in respiratory mutants and their isogenic wild-type determined at the time of glucose exhaustion in the medium

Yeast cells were grown on YEP containing 1\% glucose. Samples were harvested for metabolite extraction as described in Methods at the time of glucose exhaustion in the medium. Values reported are from duplicates taken from two independent experiments.

\begin{tabular}{|c|c|c|c|c|}
\hline \multirow[t]{2}{*}{ Strain } & \multirow[t]{2}{*}{ Genotype } & \multicolumn{3}{|c|}{$\begin{array}{c}\text { Concentration }[\mu \mathrm{mol} \text { (g dry } \\
\left.\text { mass })^{-1} \pm \mathrm{SD}\right]\end{array}$} \\
\hline & & Glc6P & ATP & ADP \\
\hline JLP48-3B & $\mathrm{RHO}^{+}$ & $0 \cdot 91 \pm 0 \cdot 10$ & $2 \cdot 35 \pm 0 \cdot 15$ & $5 \cdot 30 \pm 0 \cdot 70$ \\
\hline JF1043 & $r h o^{\circ}$ & $0.19 \pm 0.02$ & $0.84 \pm 0.05$ & $2 \cdot 05 \pm 0 \cdot 15$ \\
\hline JLP5-1A & РET309 & $1 \cdot 02 \pm 0 \cdot 15$ & $2 \cdot 85 \pm 0 \cdot 25$ & $5 \cdot 84 \pm 0 \cdot 25$ \\
\hline JF1069 & pet309 & $0 \cdot 16 \pm 0 \cdot 04$ & $0 \cdot 74 \pm 0 \cdot 15$ & $2 \cdot 15 \pm 0 \cdot 15$ \\
\hline BWG1-7A & HAP2 & $1 \cdot 06 \pm 0 \cdot 19$ & $3 \cdot 90 \pm 0 \cdot 25$ & $4 \cdot 37 \pm 0.35$ \\
\hline JO1-1A & hap2 & $0.33 \pm 0.08$ & $1 \cdot 88 \pm 0 \cdot 14$ & $6.58 \pm 0.45$ \\
\hline W303-1A & QCR9 & $0.63 \pm 0.04$ & $4 \cdot 20 \pm 0 \cdot 30$ & $3 \cdot 39 \pm 0.25$ \\
\hline JPD1 & $q c r 9$ & $0 \cdot 20 \pm 0 \cdot 05$ & $1 \cdot 70 \pm 0 \cdot 15$ & $4 \cdot 90 \pm 0 \cdot 40$ \\
\hline
\end{tabular}

\section{DISCUSSION}

The iodine-staining method has been widely used for the examination of polysaccharide storage in bacteria and yeast since differences in intracellular glycogen content could be qualitatively identified by the intensity of the brownish coloration (Carrier \& McCleskey, 1962; Chester, 1964, 1968). Despite its successful use in genetic screens devised to search for yeast mutants altered in glycogen metabolism (Thompson-Jaeger et al., 1991; Cannon et al., 1994), the reliability of this method is dependent on several parameters which have to be strictly controlled, namely the morphology of the cell (Desfarges et al., 1993), the age of the colonies (Chester, 1968), auxotrophic mutations and the composition of the growth medium (this study). The failure to control and to monitor these parameters can lead to false interpretations. A relevant illustration of this failure is the so-called glycogen deficiency of S. cerevisiae respiratory mutant strains. As discussed below, respiratory mutants are actually not impaired in glycogen synthesis, but they readily mobilize their glycogen stores during prolonged incubation in glucose-starved media. Therefore, the misleading conclusion that respiratory mutants were unable to accumulate glycogen came from the fact that iodine staining is usually performed after 2 or $3 \mathrm{~d}$ growth on agar plates, during which cells are already in a glucose-starved condition. As illustrated in this paper, a time-course iodine staining of yeast colonies is highly recommended to obtain confident data with respect to the effects of mutations on glycogen metabolism.

Our quantitative kinetic glycogen analysis showed that, contrary to previous studies (Chester, 1968; Filipak et al., 1992; Wek et al., 1992; Yang et al., 1998), mitochondrial respiratory mutants accumulated 20-50\% more glycogen than their isogenic wild-type cells during the fermentative growth phase on glucose. It is suggested that this enhanced glycogen deposition could arise from glucose that has been energetically spared by these mutants due to their inability to derepress mitochondrial functions during growth. The mechanism of this glucose flux readjustment is, however, totally unclear. Our kinetic analysis also revealed that respiratory mutants readily degraded their glycogen stores when they became starved for glucose. Hence, in contrast to the claim that a defect in respiration blocks glycogen synthesis, we showed that mitochondrial defects stimulate glycogen mobilization. Based on time-course iodine-staining experiments, the rate and the extent of glycogen degradation was apparently more potent in respirationdeficient cells cultivated on glucose minimal medium, as illustrated in $r h o^{\circ}$ cells expressing a hyperactivated form of glycogen synthase. From a physiological point of view, the mobilization of glycogen is the sole means for respiratory mutants to obtain carbon and energy in carbon-starved media as they cannot reassimilate either by-products derived from glucose fermentation or exogenous amino acids present in rich media. We also demonstrated that the capacity of respiration-deficient cells to mobilize glycogen was not suppressed by mutations in the Ras-cAMP pathway or in cyclindependent Pho85p kinase, known to negatively control glycogen metabolism (Huang et al., 1996; Timblin et al., 1996; Timblin \& Bergman, 1997; Parrou et al., 1999b). It is, however, worthy note that the mutation in these two essential nutrient-sensing pathways clearly interfered with the ability of respiration-deficient cells to efficiently degrade glycogen during prolonged incubation on minimal medium, but not on rich medium. This might be interpreted as a higher dependency of metabolic adaptations of yeast cells to these signalling pathways when the composition of the culture medium is more strict.

The sustained mobilization of glycogen during prolonged incubation of respiratory mutants in a glucosedepleted medium could not solely be explained by a less active glycogen synthase (Yang et al., 1998), since this was still observed in mutant cells expressing a constitutively activated form of this enzyme. Accordingly, a more active glycogen phosphorylase was measured in the respiration-deficient cells. In search of the mechanism which stimulates this process, it was observed, in agreement with Yang et al. (1998), that the levels of ATP and Glc6P measured at the onset of glycogen degradation dropped in respiration-deficient cells. Taking into account the fact that Glc6P is a potent activator of the dephosphorylation reaction catalysed by protein phosphatases and an inhibitor of the phosphorylation reaction catalysed by protein kinases (François \& Hers, 1988; Lin et al., 1995, 1996; Huang et al., 1997), we propose that the dramatic drop of this effector in respiration-deficient cells triggers glycogen mobilization by the activation of glycogen phosphorylase and inactivation of glycogen synthase. Hence, defects in respiration provide an additional illustration of the 
physiological function of Glc6P in the control of glycogen metabolism.

Our study on the linkage between mitochondrial function and storage carbohydrates in yeast confirmed that mitochondrial respiratory mutants were defective in trehalose synthesis (Filipak et al., 1992). Despite its inability to grow on a non-fermentable source, a gluconeogenic mutant can accumulate trehalose from glycogen mobilization because, unlike respiratory mutants, it is still able to obtain energy from the reoxidation of ethanol which is slowly taken up from the medium (François et al., 1991). Therefore, an open question is to understand how the gate for trehalose synthesis is closed to the glucosyl units derived from glycogen in respiration-deficient cells.

\section{ACKNOWLEDGEMENTS}

We thank K. Tatchell (University of Schrevenport, USA), Peter Roach (Indiana University School of Medicine, Indianapolis, USA), C. Gancedo (CSIC, Madrid) and B. Daignan-Fornier (IBGC-CNRS, Bordeaux) for generously providing yeast strains and plasmids, and Marie Ange Teste for technical assistance. This work was supported in part by the Commission of the European Union (program Cell factories no. BIO4.CT95.132).

\section{REFERENCES}

Bergmeyer, H. U. (1986). Methods in Enzymatic Analysis, 3rd edn. Weinheim, Germany: Verlag Chemie.

Cannon, J. F., Pringle, J. R., Fiechter, A. \& Khalil, M. (1994). Characterization of glycogen-deficient glc mutants of Saccharomyces cerevisiae. Genetics 136, 485-503.

Carrier, E. B. \& McCleskey, C. S. (1962). Intracellular starch formation in Corynebacteria. J Bacteriol 83, 1029-1033.

Chester, V. E. (1964). Comparative studies on dissimilation of reserve carbohydrate in four strains of Saccharomyces cerevisiae. Biochem J 92, 318-323.

Chester, V. E. (1968). Heritable glycogen-storage deficiency in yeast and its induction by ultra-violet light. J Gen Microbiol 51, 49-56.

Desfarges, L., Durrens, P., Juguelin, H., Cassagne, C., Bonneu, M. \& Aigle, M. (1993). Yeast mutants affected in viability upon starvation have a modified phospholipid composition. Yeast $\mathbf{9}$, 267-277.

Farkas, I., Hardy, T. A., Goebl, M. G. \& Roach, P. J. (1991). Two glycogen synthase isoforms in Saccharomyces cerevisiae are coded by distinct genes that are differentially controlled. J Biol Chem 266, 15602-15607.

Filipak, M., Drbot, M. A., Ireland, L. S., Singer, R. A. \& Johnston, G. C. (1992). Mitochondrial DNA loss by yeast re-entry-mutant cells conditionally unable to proliferate from stationary phase. Curr Genet 22, 471-477.

Fox, T. D., Folley, L. S., Mulero, J. J., McMullin, T. W., Thorness, P. E., Hedin, L. O. \& Costanzo, M. C. (1991). Analysis and manipulation of yeast mitochondrial genes. Methods Enzymol 194, 149-165.

François, J. \& Hers, H. G. (1988). The control of glycogen metabolism in yeast. II. A kinetic study of the two forms of glycogen synthase and of glycogen phosphorylase and an investigation of their interconversion in a cell-free extract. Eur J Biochem 174, 561-567.
François, J., Villanueva, M. E. \& Hers, H. G. (1988). The control of glycogen metabolism in yeast. I. Interconversion in vivo of glycogen synthase and glycogen phosphorylase induced by glucose, a nitrogen source or uncouplers. Eur J Biochem 174, 551-559.

François, J., Neves, M. J. \& Hers, H. G. (1991). Control of trehalose metabolism in Saccharomyces cerevisiae. Evidences for a catabolite inactivation and repression of trehalose 6-phosphate synthase and trehalose 6-phosphate phosphatase. Yeast 7, 575-587.

François, J., Thompson-Jaeger, S., Skroch, J., Zellenka, U., Spevak, W. \& Tatchell, K. (1992). GAC1 may encode a regulatory subunit for protein phosphatase type 1 in Saccharomyces cerevisiae. EMBO J 11, 87-96.

François, J., Blazquez, M. A., Arino, J. \& Gancedo, C. (1997). Storage carbohydrates in the yeast Saccharomyces cerevisiae. In Yeast Sugar Metabolism, pp. 285-311. Edited by F. K. Zimmermann \& K.-D. Entian. Lancaster, PA: Technomics Publishing.

Gonzalez, B., François, J. \& Renaud, M. (1997). A rapid and reliable method for metabolite extraction in yeast using boiling buffered ethanol. Yeast 13, 1347-1356.

Guthrie, C. \& Fink, G. R. (1991). Guide to yeast genetics and molecular biology. Methods Enzymol 194, 3-37.

Hardy, T. A. \& Roach, P. J. (1993). Control of yeast glycogen synthase-2 by COOH-terminal phosphorylation. J Biol Chem 268, 23799-23805.

Hardy, T. A., Huang, D. \& Roach, P. J. (1994). Interactions between cAMP-dependent and SNF1 protein kinase in the control of glycogen accumulation in Saccharomyces cerevisiae. J Biol Chem 269, 27907-27913.

Huang, D., Farkas, I. \& Roach, P. J. (1996). Pho85p, a cyclindependent protein kinase, and Snf1p protein kinase act antagonistically to control glycogen accumulation in Saccharomyces cerevisiae. Mol Cell Biol 16, 4357-4365.

Huang, D., Wilson, W. A. \& Roach, P. J. (1997). Glc6P control of glycogen synthase phosphorylation in yeast. J Biol Chem 272, 22495-22501.

Hwang, P. K., Stugendreich, S. \& Fletterick, R. J. (1989). Molecular analysis of GPH1, the gene encoding glycogen phosphorylase in Saccharomyces cerevisiae. Mol Cell Biol 9, 1659-1666.

Lillie, S. H. \& Pringle, J. R. (1980). Reserve carbohydrate metabolism in Saccharomyces cerevisiae: responses to nutrient limitation. J Bacteriol 141, 1384-1394.

Lin, K., Hwang, P. K. \& Fletterick, R. J. (1995). Mechanism of regulation in yeast glycogen phosphorylase. J Biol Chem 270, 26833-26839.

Lin, K., Rath, V. L., Dai, S. C., Fletterick, R. J. \& Hwang, P. K. (1996). A protein phosphorylation switch at the conserved allosteric site in GP. Science 273, 1539-1541.

Manthey, G. M. \& McEwen, J. E. (1995). The product of the nuclear gene PET309 is required for translation of mature mRNA and stability or production of intron-containing RNAs derived from the mitochondrial COX1 locus of Saccharomyces cerevisiae. EMBO J 14, 4031-4043.

Ni, H.-T. \& Laporte, D. C. (1995). Response of a yeast glycogen synthase gene to stress. Mol Microbiol 16, 1197-1205.

Parrou, J. L. \& François, J. (1997). A simplified procedure for a rapid and reliable assay of both glycogen and trehalose in whole yeast cells. Anal Biochem 248, 186-188. 
Parrou, J. L., Teste, M. A. \& François, J. (1997). Effects of various types of stress on the metabolism of reserve carbohydrates in Saccharomyces cerevisiae: genetic evidence for a stress-induced recycling of glycogen and trehalose. Microbiology 143, 1891-1900.

Parrou, J. L., Enjalbert, B., Plourde, L., Bauche, A., Gonzalez, B. \& François, J. (1999a). Dynamic responses of reserve carbohydrate metabolism under carbon and nitrogen limitations in Saccharomyces cerevisiae. Yeast 15, 191-203.

Parrou, J. L., Enjalbert, B. \& François, J. (1999b). STRE and cAMPindependent transcriptional induction of Saccharomyces cerevisiae GSY2 encoding glycogen synthase during diauxic growth on glucose. Yeast 15, 1471-1484.

Rose, M. \& Botstein, D. (1983). Construction and use of gene fusions lacZ ( $\beta$-galactosidase) which are expressed in yeast. Methods Enzymol 101, 167-180.

Schiestl, R. H. \& Gietz, R. D. (1989). High efficiency transformation of intact yeast cells using single stranded nucleic acids as carrier. Curr Genet 16, 339-346.

Skroch-Stuart, J., Frederick, D., Varner, C. M. \& Tatchell, K. (1994). The mutant type 1 protein phosphatase encoded by $g l c 7-1$ from Saccharomyces cerevisiae fails to interact productively with the GAC1-encoded regulatory subunit. Mol Cell Biol 14, 896-905.

Timblin, B. \& Bergman, L. W. (1997). Elevated expression of stress response genes resulting from deletion of the PHO85 gene. Mol Microbiol 26, 981-990.

Timblin, B., Tatchell, K. \& Bergman, L. W. (1996). Deletion of the gene encoding the cyclin-dependent protein kinase Pho85 alters glycogen metabolism in Saccharomyces cerevisiae. Genetics 143 57-66.

Thompson-Jaeger, S., François, J., Gaughran, J. \& Tatchell, K. (1991). Deletion of SNF1 affects the nutrient response of yeast and resembles mutations which activate the adenylate cyclase pathway. Genetics 129, 697-706.

Thon, V. J., Vigneron-Lesens, C., Marianne-Pepin, T., Montreuil, J., Decq, A., Rachez, C., Ball, S. G. \& Cannon, J. F. (1992). Coordinate regulation of glycogen metabolism in yeast Saccharomyces cerevisiae. J Biol Chem 267, 15224-15228.

Tzagaloff, A. \& Dieckmann, C. L. (1990). PET genes in Saccharomyces cerevisiae. Microbiol Rev 54, 211-225.

Wach, A., Brachat, A., Pölhmann, R. \& Philippsen, P. (1994). New heterologous modules for classical or PCR-based gene disruptions in Saccharomyces cerevisiae. Yeast 10, 1793-1808.

Wek, R. C., Cannon, J. F., Dever, T. E. \& Hinnebusch, A. G. (1992). Truncated protein phosphatase GLC7 restores translational activation of GCN4 expression in yeast mutants defective for the eIF-2 $\alpha$ kinase GCN2. Mol Cell Biol 12, 5700-5710.

Wilson, W. A., Mahrenholz, A. M. \& Roach, P. J. (1999). Substrate targeting of the yeast cyclin-dependent kinase Pho85p by cyclin Pcl10p. Mol Cell Biol 19, 7020-7030.

Yang, R., Chun, K. T. \& Wek, R. C. (1998). Mitochondrial respiratory mutants in yeast inhibit glycogen accumulation by blocking activation of glycogen synthase. J Biol Chem 273, 31337-31344. 